\title{
Cerebral Hyperperfusion Syndrome following the Excision of a Mycotic Aneurysm with Superficial Temporal Artery-to-Middle Cerebral Artery Bypass: Case Report
}

\author{
Hidemichi ITO, ${ }^{1}$ Yuichiro TANAKA, ${ }^{1}$ Taigen SASE, ${ }^{1}$ Masashi UCHIDA, ${ }^{1}$ \\ Yasuyuki YOSHIDA, ${ }^{1}$ Yohtaro SAKAKIBARA, ${ }^{1}$ and Takuo HASHIMOTO ${ }^{1}$ \\ ${ }^{1}$ Department of Neurosurgery, St. Marianna University School of Medicine, \\ Kawasaki, Kanagawa
}

\begin{abstract}
The authors report a rare case of cerebral hyperperfusion syndrome (HPS) following the excision of a mycotic aneurysm with superficial temporal artery-to-middle cerebral artery (STA-MCA) bypass. A 74-yearold woman with infective endocarditis presented with progressive cerebral infarction and subarachnoid hemorrhage due to a mycotic aneurysm, which was excised with a STA-MCA bypass. Postoperatively, the patient developed HPS that was considered to be exacerbated by a previous ischemic event. Therefore, cerebral hemodynamics should be evaluated before bypass surgery to prevent subsequent hyperperfusion.
\end{abstract}

Key words: bypass, cerebral blood flow, hyperperfusion syndrome, mycotic aneurysm, single-photon emission computed tomography

\section{Introduction}

Intracranial lesions associated with infective endocarditis include mycotic aneurysms, cerebral infarctions, encephalitis, meningitis, and brain abscesses due to septic vegetation-derived emboli. ${ }^{1)}$ Treatment for mycotic aneurysms consists of antibiotics and surgical interventions to prevent bleeding. Utilization of superficial temporal artery-to-middle cerebral artery (STA-MCA) bypass for mycotic aneurysm treatment has also been reported. ${ }^{2,3)}$ Here, we report a rare case of hyperperfusion syndrome (HPS) following excision of a mycotic aneurysm with STA-MCA bypass. Although HPS after bypass surgery for intracranial atherosclerotic disease or moyamoya disease has been reported previously, HPS after surgery for mycotic aneurysms has not yet been described. ${ }^{4-6)}$

\section{Case Report}

The patient was a right-handed 74-year-old woman with a history of chronic heart failure and dental caries. Approximately 3 months after the initiation of conservative treatment, she was found unconscious in a bathtub

Received January 23, 2013; Accepted April 8, 2013 and was referred to our hospital.

On admission, she presented with slightly disturbed consciousness but no localized neurological deficits. Plain-film thoracic radiography revealed heart failure, and echocardiography indicated severe mitral regurgitation (MR) associated with valve vegetation. Streptococcus mutans was detected in patient's blood cultures.

\section{Neuroradiological findings}

Computed tomography (CT) revealed a low-density lesion, and diffusion-weighted imaging (DWI) indicated high-intensity signals in the left occipital lobe. Magnetic resonance angiography (MRA) showed occlusion of the left posterior cerebral artery (Fig. 1). On the basis of these three findings, the patient was comprehensively diagnosed with heart failure due to MR and cardiogenic cerebral embolism due to infective endocarditis vegetations.

\section{Clinical course after admission}

The patient gradually regained consciousness, and her cardiac function improved after antibiotic administration and circulatory management. However, she developed generalized convulsions on day 30 of hospitalization. Magnetic resonance imaging (MRI) revealed multiple new cerebral infarctions in the region of the left middle cerebral artery (MCA), and a small amount of subarachnoid 

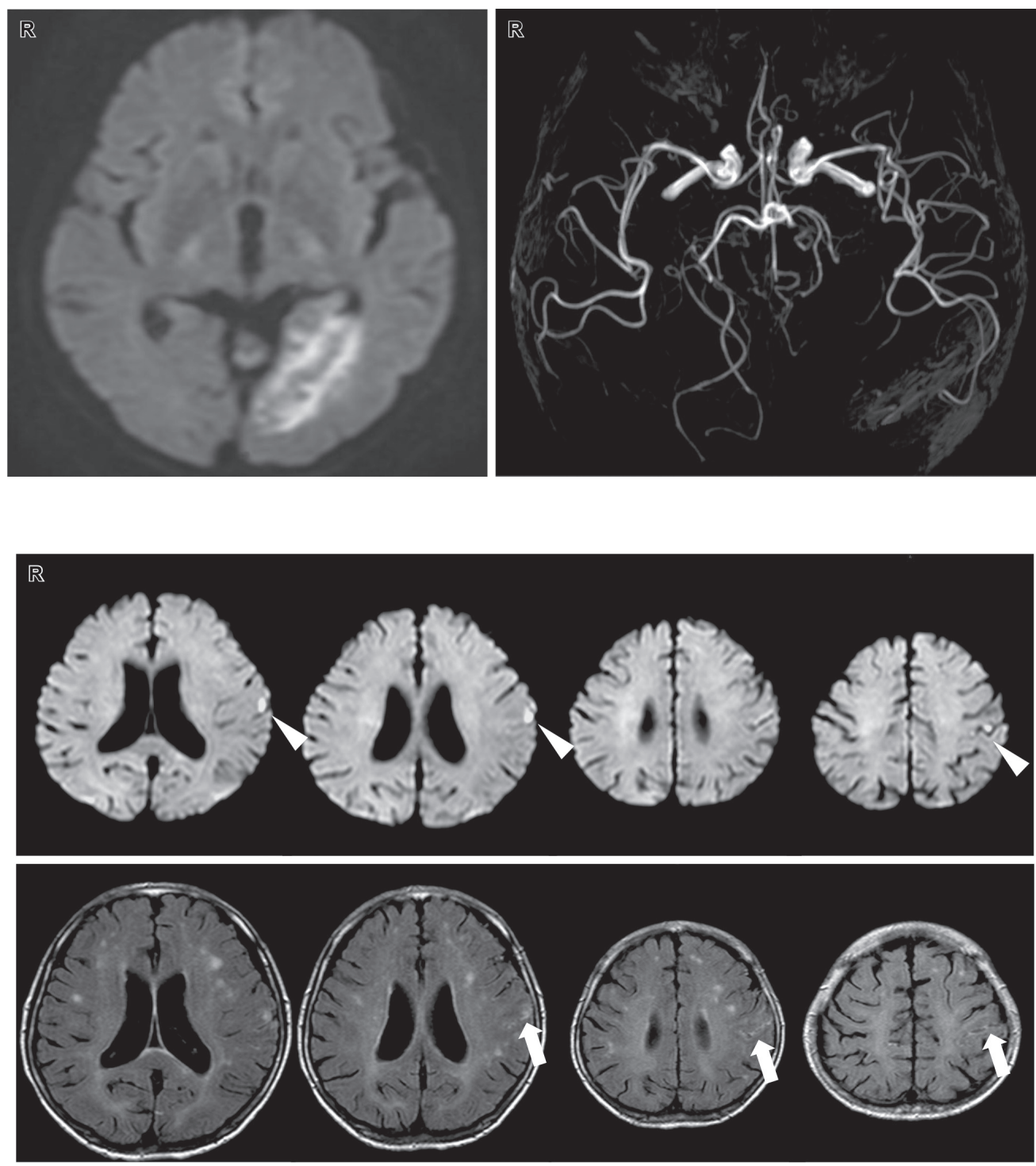

Fig. 1 Diffusion-weighted imaging performed at admission (left) showed an acute cerebral infarction in the left occipital lobe, and magnetic resonance angiography (right) indicated left posterior cerebral artery occlusion.
Fig. 2 MRI performed on day 30 of hospitalization. Upper section: DWI revealed a diffuse acute cerebral infarction (arrowhead). Lower section: FLAIR images showed a small amount of SAH along the left central sulcus (arrow). DWI: diffusion-weighted imaging, FLAIR: fluid attenuated inversion recovery, MRI: magnetic resonance imaging, SAH: subarachnoid hemorrhage. hemorrhage (SAH; Fig. 2). Cerebral angiography demonstrated an irregular aneurysm $4 \mathrm{~mm}$ in diameter at the bifurcation of the left parietal artery (Fig. 3), and she was diagnosed with a mycotic aneurysm. Furthermore, narrowing of the parent and bifurcating vessels (anterior parietal and posterior parietal arteries) was found, which suggested vasospasm or vasculitis. The patient's MR was severe and indicated an early valve replacement. Surgical treatment for the mycotic aneurysmwas prioritized to avoid rebleeding because valve replacement surgery requires systemic heparinization.

\section{Surgery}

The surgery was conducted on the 58th day of hospitalization. Her cardiocirculatory dynamics were stable, and inflammation had subsided. The serum C-reactive protein level was negative at that time. Because the dominant hemisphere was involved, the therapeutic strategy included excision of the mycotic aneurysm with STA-MCA bypass. Parietal craniotomy was performed on the left side. Upon opening the dura mater, a red-brown aneurysm was found at the surface of the brain. The dura mater and aneurysm were strongly adherent, suggesting inflammation. The STA was anastomosed to the posterior parietal artery, which measured approximately $1 \mathrm{~mm}$ in diameter, and the mycotic aneurysm was resected after parent vessel ligation (Fig. 4). The anterior parietal artery was ligated because it was markedly narrowed. Total vascular clamping duration was $21 \mathrm{~min}$. Pathological analysis of the aneurysm revealed rupture of vascular wall elastic fibers accompanied by calcification. An organized thrombus was present in the lumen, but no bacteria were detected. These findings led to the diagnosis of false aneurysm with chronic inflammation. 

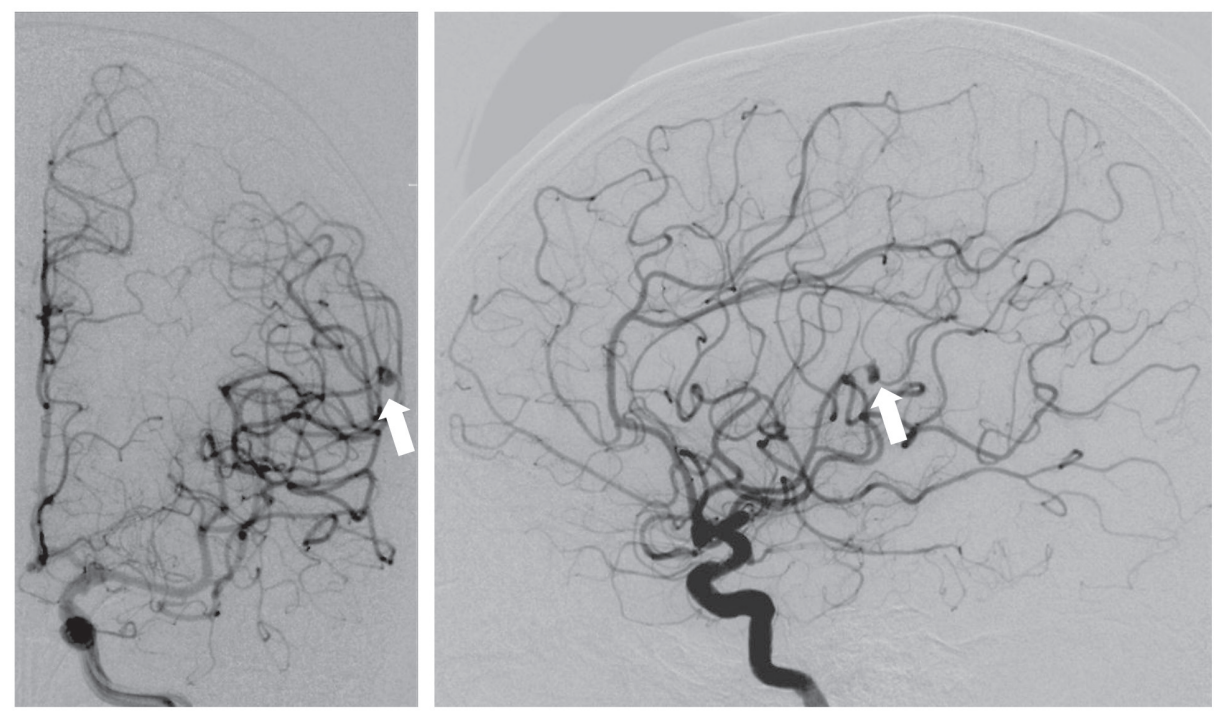

Fig. 3 Angiography was performed on day 30 of hospitalization. An irregular 4-mm aneurysm was found at the bifurcation of the left parietal artery (arrow, left: anteroposterior view; right: lateral view).
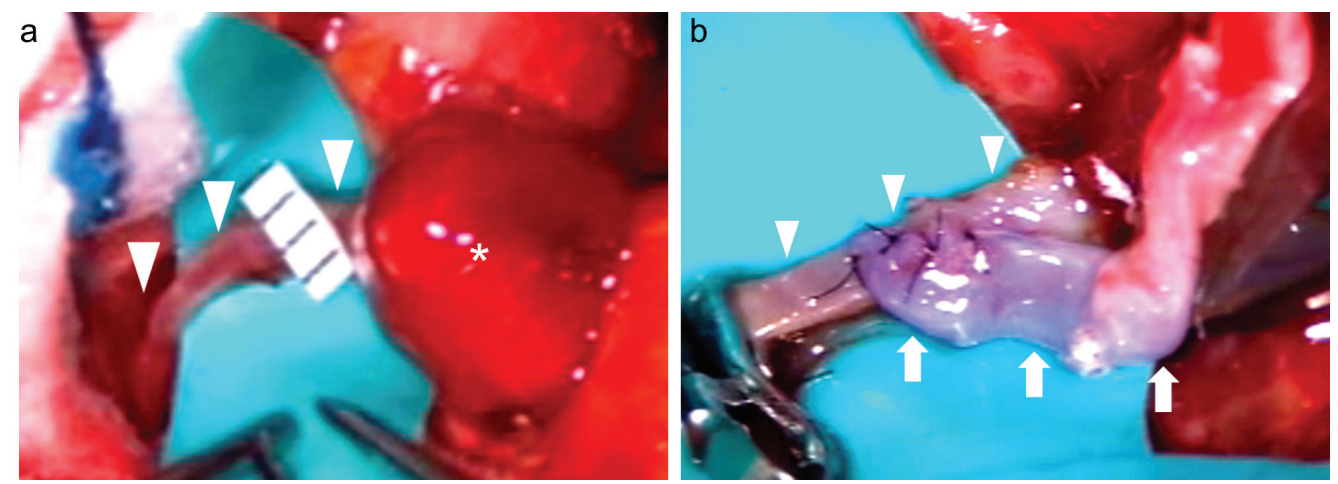

Fig. 4 a: Intraoperative photograph showing a mycotic aneurysm (asterisk) and the posterior parietal artery (arrowhead). The aneurysm was reddish-brown, and the posterior parietal artery measured approximately $1 \mathrm{~mm}$ in diameter. b: The STA was anastomosed to the posterior parietal artery. STA: superficial temporal artery.

\section{Postoperative course}

No neurological deficits were observed, and postoperative CT showed no abnormal lesions other than the initial infarction. On the second day after surgery, the patient developed aphasia and right upper limb paralysis, but CT showed no abnormalities. MRA showed a dilated left MCA, and $N$-isopropyl- $p$-[ $\left.{ }^{123} \mathrm{I}\right]$-iodoamphetamine ( ${ }^{123} \mathrm{I}$-IMP) single-photon emission CT (SPECT) revealed increased blood flow in the left temporal and parietal lobes. These findings led to the diagnosis of HPS (Fig. 5a). Edaravone was administered after the patient's systolic blood pressure was controlled at approximately $90-100 \mathrm{mmHg}$ with a sedative agent. Because her mitral valve dysfunction was not yet treated, her blood pressure was not too high. MRA performed 9 days and 1 month after the surgery showed that the dilated MCA was recovering its original diameter. The hyperperfusion detected on ${ }^{123}$ I-IMP SPECT resolved gradually, but blood flow in the left temporal and parietal lobes had decreased (Fig. 5b, c). ${ }^{123}$ I-IMP SPECT performed 3 months after surgery revealed little change, and MRI showed no new cerebral infarctions. After the HPS resolved, the patient without any neurological defi- cits underwent mitral valve replacement and received a pacemaker and was discharged from the hospital a month after cardiac surgery. ${ }^{123}$ I-IMP SPECT and CT performed 6 months after surgery did not reveal any new lesions, and her postoperative course remained uneventful 1 year after surgery.

\section{Discussion}

Infective endocarditis is a septic disease characterized by bacterial vegetations in the endocardium, heart valves, and intimas of large blood vessels. The clinical manifestations of infective endocarditis include bacteremia, impaired cardiac function, and embolisms in multiple organs. ${ }^{1)}$ Intracranial lesions, including mycotic aneurysms, cerebral infarctions, encephalitis, meningitis, and brain abscesses due to septic embolisms from vegetations, are found in $20-50 \%$ of cases. The morbidity rate associated with infective endocarditis and mycotic aneurysms is $3-15 \%$. Because the prognosis for ruptured mycotic aneurysms is extremely poor, proper treatment is of the utmost importance. ${ }^{7)}$ With the exception of emergency hematoma 

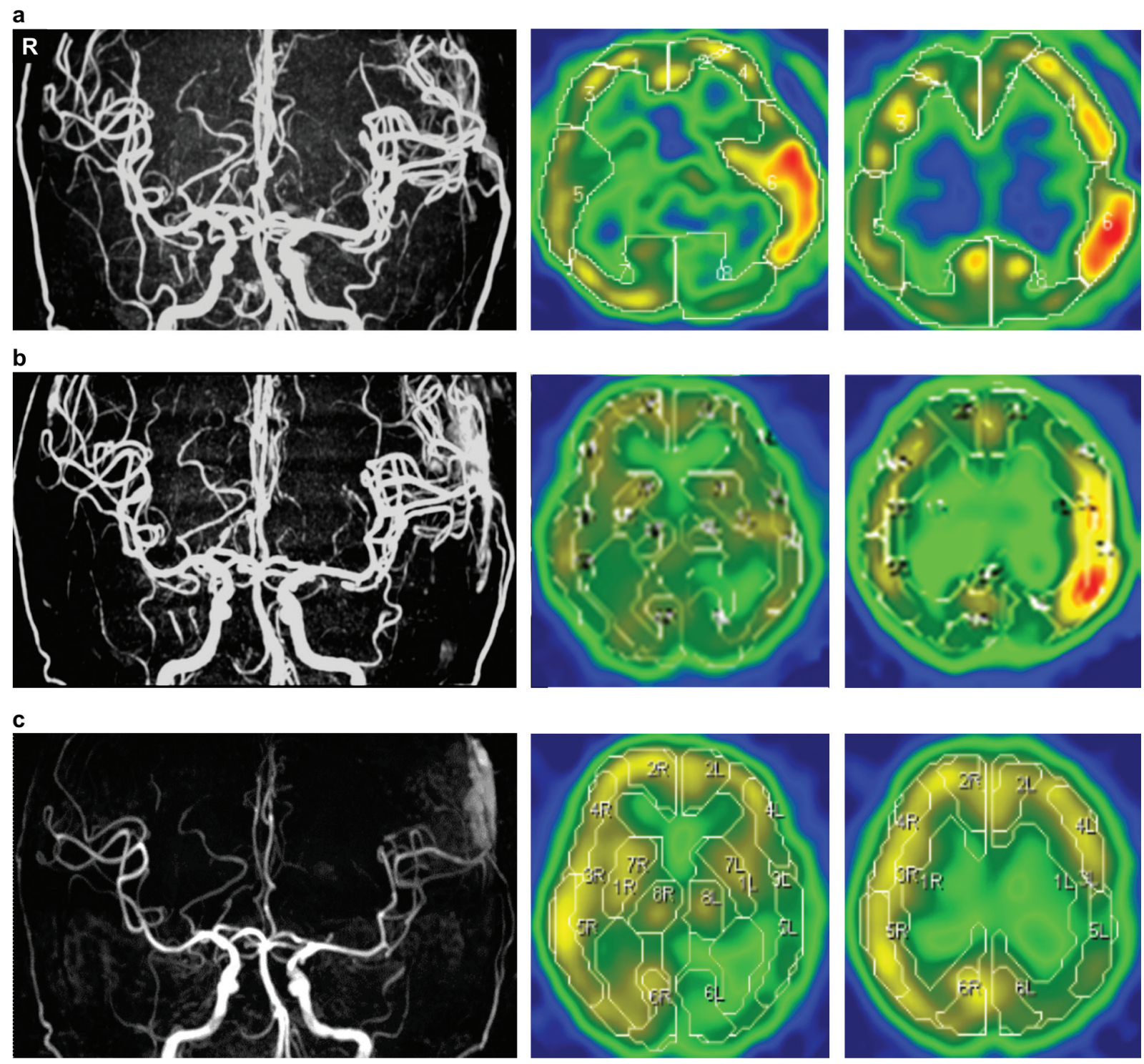

Fig. 5 a: MRA performed 2 days after surgery revealed left MCA dilation, and $N$-isopropyl-p-[ $\left.{ }^{123} I\right]$-iodoamphetamine $\left.{ }^{(23} I-I M P\right)$ SPECT showed hyperperfusion in the left temporal and parietal lobes. b: MRA performed 9 days after surgery showed that the dilated MCA was recovering its original diameter; the hyperperfusion detected on ${ }^{123}$ I-IMP SPECT resolved gradually. c: MRA performed 1 month after surgery showed no dilation of the left MCA, and ${ }^{123}$ I-IMP SPECT showed resolution of the hyper- and hypoperfusion in the left temporal and parietal lobes, which had previously shown hyperperfusion. MCA: middle cerebral artery, MRA: magnetic resonance angiography, SPECT: single-photon emission computed tomography.

removal, mycotic aneurysm treatment is conservative and consists of continuous antibiotic administration for several weeks, which usually results in spontaneous regression of the aneurysm. Surgical treatment is recommended if the aneurysm remains unchanged or increases in volume despite antibiotic treatment. ${ }^{8)}$ However, there is no standard therapeutic strategy, and the indication and optimal timing of surgery, as well as the modalities of treatment are still debatable., ${ }^{2,8,9)}$

The patient described here was resistant to the 30-day antibiotic treatment and developed an SAH. Furthermore, the patient's heart failure due to MR necessitated valve replacement. Because systemic heparinization is required during open-heart surgery, there was a risk of intracranial hemorrhage, and craniotomy was performed first. The aneurysm had no clearly visible neck, and the adjacent vessels had narrowed; therefore, neck clipping surgery and intra-aneurysmal coil embolization were unsuitable treatment options. Moreover, the aneurysm was located at the bifurcation of the left 
parietal artery, and simple excision of the aneurysm could have resulted in cerebral ischemia. Therefore, resection with STA-MCA bypass was performed. Although bypass surgery is not often performed to treat mycotic aneurysms, some previous reports have indicated that this method is effective. ${ }^{2,3)}$

The incidence of HPS is $0.4-1.9 \%$ in carotid endarterectomies, $5.0-6.0 \%$ in extracranial-intracranial bypass surgeries to treat intracranial atherosclerotic disease, and $12.0-21.5 \%$ in extracranial-intracranial bypass surgeries to treat moyamoya disease. ${ }^{4-6,10)}$ However, HPS after extracranial-intracranial bypass surgery for the treatment of mycotic aneurysm is rare and has not been reported previously. The causes of HPS include autoregulation disorders after chronic hypoperfusion due to severe vessel stenosis or poor collateral circulation, ${ }^{11)}$ temporary interruption of blood flow during surgery, ${ }^{122}$ and a history of cerebral ischemia due to microemboli. ${ }^{13)}$ Furthermore, increased levels of free radicals can damage blood vessel intimas. ${ }^{14)}$ In the present case, although cerebral blood flow was not evaluated preoperatively, chronic ischemia was suspected because the patient showed hypotension due to severe MR and heart failure, microcirculatory disturbances due to diffuse cerebral infarction in the MCA, poor collateral circulation due to posterior cerebral artery occlusion, and vasospasm or vasculitis around the aneurysm. Fujimura et al. have reported that matrix metalloproteinase-9 contributes directly to hemorrhagic complication after extracranial-intracranial bypass surgery for moyamoya disease. ${ }^{15}$ Matrix metalloproteinase-9 is a biochemical marker of blood-brain barrier disruption. Because the operative findings and specimens confirmed chronic inflammation, malfunction of the blood-brain barrier of the vessels around a mycotic aneurysm could have triggered hyperperfusion.

Was STA-MCA bypass necessary for this patient? Mycotic aneurysms are generally characterized by an irregular shape, absence of a clearly visible neck, and fragile aneurysms and parent arteries ${ }^{8}{ }^{8}$ In the present case, the lesions were peripheral, and the adjacent vessels had narrowed, which may have made anastomosis difficult during a lengthy surgery. This could be considered a refractory case, in which the surgery may have exacerbated the inflammation, and the parent artery itself would have likely been occluded. ${ }^{2)}$ Although the necessity of the bypass surgery for a peripheral vessel disease was debatable, we elected to use the method because dominant cerebral blood flow had not been evaluated. Typically, an occlusion test or a provocative test should be performed before hemostatic surgery, ${ }^{3,16)}$ and it would be probably safe if the results of these tests are negative. However, advancing a catheter is difficult when the targeted blood vessel is located in the periphery. Furthermore, catheterizing a blood vessel that has been weakened by inflammation is risky; therefore, these tests were not performed. Accordingly, there was no incidence of a postoperative cerebral infarction despite the sacrifice of the anterior parietal artery. This may have been because the affected region was located adjacent to the posterior parietal artery, which had abundant blood flow after bypass surgery.

Although most mycotic aneurysms are located in peripheral areas, the best treatment is neck-clipping surgery once inflammation has subsided. If this is not feasible, excision of the parent vessel should be performed. In such cases, this procedure should be combined with bypass surgery, and blood flow must be preserved as much as possible. Preoperative evaluation of cerebral blood flow is useful for selecting the proper surgical method and postoperative management, as well as for predicting the possibility of HPS.

\section{Conclusion}

We described a patient who developed HPS following STA-MCA bypass surgery for a ruptured mycotic aneurysm, which was complicated by an infective endocarditis. The HPS might have resulted from chronic hypoperfusion, which was present before the surgery. The patient's infective endocarditis pathogenesis included two aspects: cerebralischemia and hemorrhage, underscoring the importance of preoperative evaluation of cerebral blood flow for selecting the proper surgical method and postoperative management.

\section{Conflicts of Interest Disclosure}

The authors have no conflicts of interest to declare.

\section{References}

1) Chu VH, Cabell CH, Benjamin DK, Kuniholm EF, Fowler VG, Engemann J, Sexton DJ, Corey GR, Wang A: Early predictors of in-hospital death in infective endocarditis. Circulation 109: 1745-1749, 2004

2) Chun JY, Smith W, Halbach VV, Higashida RT, Wilson CB, Lawton MT: Current multimodality management of infectious intracranial aneurysms. Neurosurgery 48: 1203-1213; discussion 1213-1214, 2001

3) Day AL: Extracranial-intracranial bypass grafting in the surgical treatment of bacterial aneurysms: report of two cases. Neurosurgery 9: 583-588, 1981

4) Fujimura M, Shimizu H, Inoue $T$, Mugikura S, Saito A, Tominaga T: Significance of focal cerebral hyperperfusion as a cause of transient neurologic deterioration after extracranial-intracranial bypass for moyamoya disease: comparative study with non-moyamoya patients using $N$-isopropyl-p-[123I]iodoamphetamine single-photon emission computed tomography. Neurosurgery 68: 957-964; discussion 964-965, 2011

5) Hayashi K, Horie N, Suyama K, Nagata I: Incidence and clinical features of symptomatic cerebral hyperperfusion 
syndrome after vascular reconstruction. World Neurosurg 78: 447-454, 2012

6) Yamaguchi K, Kawamata T, Kawashima A, Hori T, Okada Y: Incidence and predictive factors of cerebral hyperperfusion after extracranial-intracranial bypass for occlusive cerebrovascular diseases. Neurosurgery 67: 1548-1554; discussion 1554, 2010

7) Salgado AV, Furlan AJ, Keys TF, Nichols TR, Beck GJ: Neurologic complications of endocarditis: a 12-year experience. Neurology 39: 173-178, 1989

8) Bingham WF: Treatment of mycotic intracranial aneurysms. J Neurosurg 46: 428-437, 1977

9) Phuong LK, Link M, Wijdicks E: Management of intracranial infectious aneurysms: a series of 16 cases. Neurosurgery 51: 1145-1151; discussion 1151-1152, 2002

10) Ogasawara K, Sakai N, Kuroiwa T, Hosoda K, Iihara K, Toyoda K, Sakai C, Nagata I, Ogawa A, Japanese Society for Treatment at Neck in Cerebrovascular Disease Study Group: Intracranial hemorrhage associated with cerebral hyperperfusion syndrome following carotid endarterectomy and carotid artery stenting: retrospective review of 4494 patients. J Neurosurg 107: 1130-1136, 2007

11) Ogasawara K, Yukawa H, Kobayashi M, Mikami C, Konno $\mathrm{H}$, Terasaki K, Inoue T, Ogawa A: Prediction and monitoring of cerebral hyperperfusion after carotid endarterectomy by using single-photon emission computerized tomography scanning. J Neurosurg 99: 504-510, 2003

12) Komoribayashi N, Ogasawara K, Kobayashi M, Saitoh H, Terasaki K, Inoue T, Ogawa A: Cerebral hyperperfusion after carotid endarterectomy is associated with preoperative hemodynamic impairment and intraoperative cerebral ischemia. J Cereb Blood Flow Metab 26: 878-884, 2006

13) Roh HG, Byun HS, Ryoo JW, Na DG, Moon WJ, Lee BB, Kim DI: Prospective analysis of cerebral infarction after carotid endarterectomy and carotid artery stent placement by using diffusion-weighted imaging. AJNR Am J Neuroradiol 26: 376-384, 2005

14) Ogasawara K, Inoue T, Kobayashi M, Endo H, Fukuda T, Ogawa A: Pretreatment with the free radical scavenger edaravone prevents cerebral hyperperfusion after carotid endarterectomy. Neurosurgery 55: 1060-1067, 2004

15) Fujimura $M$, Watanabe $M$, Narisawa $A$, Shimizu H, Tominaga $T$ : Increased expression of serum Matrix Metalloproteinase-9 in patients with moyamoya disease. Surg Neurol 72: 476-480; discussion 480, 2009

16) Linskey ME, Jungreis CA, Yonas H, Hirsch WL Jr, Sekhar LN, Horton JA, Janosky JE: Stroke risk after abrupt internal carotid artery sacrifice: accuracy of preoperative assessment with balloon test occlusion and stable xenon-enhanced CT. AJNR Am J Neuroradiol 15: 829-843, 1994

Address reprint requests to: Hidemichi Ito, MD, Department of Neurosurgery, St. Marianna University School of Medicine, 2-16-1 Sugao, Miyamae-ku, Kawasaki, Kanagawa 216-8511, Japan.

e-mail: hdmcito@marianna-u.ac.jp 